\title{
Excitation-Transfer Plasmonic Nanosensors based on Dynamical Phase Transitions.
}

\author{
Raúl A. Bustos-Marún, ${ }^{* \dagger}$ Eduardo A. Coronado, ${ }^{\ddagger}$ and Horacio M. Pastawski ${ }^{\dagger}$ \\ IFEG (CONICET) and Facultad de Matemática Astronomía y Física, Universidad Nacional de \\ Córdoba, ,5000, Córdoba, Argentina, and INFIQC (CONICET) and Facultad de Ciencias \\ Químicas, Universidad Nacional de Córdoba, 5000, Córdoba, Argentina \\ E-mail: rbustos@famaf.unc.edu.ar
}

\footnotetext{
${ }^{*}$ To whom correspondence should be addressed

${ }^{\dagger}$ IFEG and FAMAF, UNC

${ }^{\ddagger}$ INFIQC and FCQ, UNC
} 


\begin{abstract}
Dynamical Phase transitions (DPTs) describe the abrupt change in the dynamical properties of open systems when a single control parameter is slightly modified. Recently we found that this phenomenon is also present in a simple model of a linear array of metallic nanoparticles (NPs) in the form of a localized-delocalized DPT. In this work we show how to take advantage of DPTs in order to design a new kind of plasmonic sensor which should own some unique characteristics. For example, if it were used as plasmon ruler it would not follow the so called universal plasmon ruler equation [Nano Letters 2007, 7, 2080-2088], exhibiting instead an on-off switching feature. This basically means that a signal should only be observed when the control/measured parameter, i.e. a distance in the case of plasmon rulers, has a very precise and pre-determined value. Here, we demonstrate their feasibility and unique characteristics, showing that they combine high sensitivity with this on-off switching feature in terms of different distances and local dielectric constants. This property has the potentiality to be used in the design of new plasmonic devices such as plasmonic circuits activated only under certain environmental conditions.
\end{abstract}

Keywords: plasmonics, one dimensional arrays, open systems, localization, plasmon rulers.

\title{
INTRODUCTION
}

The study of localized surface plasmon (LSP) resonances over the last couple of decades has led to great advances in several areas of science and technology. Probably, its most significant application is its use in nanosensors. ${ }^{1-26}$ Most of the strategies used to turn a plasmonic device into a sensor are based on the shift of the resonant position of the LSP $1-16$ or in exploiting hot spots to increase spectroscopic signals. ${ }^{23}-26$ In this respect, there is a growing interest in using interacting plasmonic systems to measure distances at the nanoscale. These devices are known as nanorulers and they are finding interesting applications in several fields such as biology. $\underline{8}$ Most of the proposed plasmonic rulers work by the same principle, the shift of a resonance induced by the variation of a distance. As noticed originally by Jain et. al., ${ }^{9}$ the frequency shift of the LSP resonance follows 
an almost universal exponential or quasi-exponential law with respect to the separation between NPs. This behavior is known as the universal plasmon ruler equation (UPRE) and was observed in simulations and experiments using NPs of different size and shape, $\stackrel{9-11}{\underline{m}}$ core-shell NPs, $\stackrel{15}{\underline{a}}$ and even two dimensional arrays of NPs. $\stackrel{16}{ }$ There are some deviations of the UPRE due to retardation effects or the effects of higher order multipoles, but they can usually be corrected by empirical fittings. $\stackrel{7}{-}$ Only when NPs are almost touching each other, there are some deviations, nonfitable to exponential, of the UPRE. $\stackrel{13,14}{ }$ There is another kind of plasmon rulers that is now being actively investigated. They are based on a different principle, the fluorescence resonance energy transfer (FRET). Its working principle is the resonant transference of excitation from a fluorescent molecule to a nearby NP. The closer to fluorophore to the NP, the greater the photoluminescence quenching. This effect has been successfully used as a nanorulers in several experimental examples. $\underline{17}-\underline{22}$

Collective effects induced by LSP couplings in NPs arrays can give rise to other phenomena and new potential applications. ${ }^{27-41}$ One example is the use of Fano resonances to design plasmonic sensors. $\frac{3}{3}$ In this case, the shift of very sharp peaks or valleys in the extinction spectrum, consequence of collective effects of interacting NPs, have been proposed to measure distances or the dielectric constant of the surrounding medium. $\frac{3}{-}$ More exotic applications of NPs coupling are also possible and many of them are now being actively explored, such as clocking by metamaterials or plasmonics circuitry. $\underline{43,44}$

Although, linear arrays of metallic NPs have been proposed as potential waveguides to transfer massive amounts of information at the nanoscale, $\stackrel{45}{=}$ nowadays it is clear that high damping factors would impose severe limits to that and most of the effort in this field is being put on overcoming this limitation. However, one important and very general feature of 1-D systems, which seems to have been overlooked in plasmonics, is the extreme sensitivity of their dynamical properties to slight changes of their parameters. In this respect, we have previously studied the plasmonics energy transfer from a locally excited NP (LE-NP), the 0th NP in the scheme shown in 1 , to the interior of a semi-infinite NP chain. $\underline{46}^{6}$ This system presents a form of dynamical phase transition (DPT), a phenomenon that is currently attracting interest as source of novel effects on various 
fields. $\underline{47}-51$ Basically a DPT in this context means that by sightly moving a single parameter, such as the shape of the NPs or the distance between them, the system undergoes an abrupt transition from transferring all the injected excitation to the interior of the NP array (a delocalized state), to keeping all the external excitation in the NPs closer to the excitation point (a localized state). $\underline{46}$ The aim of this work is to exploit this basic property into a realistic setting, in order to design a new kind of excitation-transfer plasmonic nanosensor (ETPS).

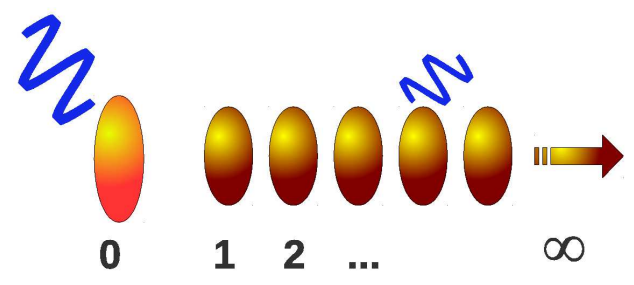

Figure 1: Scheme of the system studied. An external source locally excites the LSP of a NP which is coupled to a waveguide where detection takes place. The arrow on the right indicates that the array is infinite in this direction.

\section{THEORY}

The system studied, depicted in 1 is modeled using the coupled dipole approximation. $\underline{29-33,36-42,45,46}$ In this model, each $i^{\text {th }}$-NP is described by a dipole $P_{i}$ induced by the electric field produced by the other dipoles, $E_{i, j}$, and the external source, $E_{i}^{(\mathrm{ext})}$. We assume a generic ellipsoidal shape for the

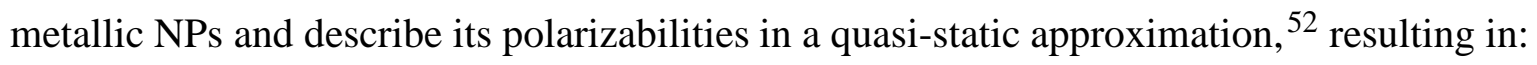

$$
P_{i}=\frac{\varepsilon_{0} \mathscr{V}_{i}\left(\varepsilon_{i}-\varepsilon_{m, i}\right)}{\left[\varepsilon_{m, i}+L_{i}\left(\varepsilon_{i}-\varepsilon_{m, i}\right)\right]}\left(E_{i}^{(\mathrm{ext})}+\sum_{j \neq i} E_{i, j}\right)
$$

where $\mathscr{V}$ is the volume, $L$ is a geometrical factor that depends on the shape of the NP and the direction of $E, \varepsilon_{0}$ is the free space permittivity, and $\varepsilon_{m, i}$ is the dielectric constant of the host medium around the $i$-th NP. The dielectric constants $\varepsilon_{i}$ of the NPs are described by a Drude-Sommerfeld model: $\varepsilon=\varepsilon_{\infty}-\frac{\omega_{\mathrm{p}}^{2}}{\left(\omega^{2}+i \omega \eta\right)}$, where $\omega_{\mathrm{p}}$ is the plasmon frequency, $\eta$ is the electronic damping factor, and $\varepsilon_{\infty}$ is a material dependent constant that take into account the contribution of the bound 
electrons to the polarizability. The LSP oscillations over each NP can only be transverse $(T)$ or longitudinal $(L)$ to the axis of the linear array and we assume, for simplicity, that the ellipsoids axes are aligned with respect to these $T$ and $L$ directions. If the excitation wavelength is large compared with the separation between NPs, $E_{i, j}$ can be taken in the near field approximation, $E_{i, j}=\frac{-\gamma^{T, L} P_{j}}{4 \pi \varepsilon_{0} \varepsilon_{m} d_{i, j}^{3}}$, where $\gamma^{T}=1, \gamma^{L}=-2$ and $d$ is the distance between NPs. Taking into account these considerations, $P_{i}$ and $E_{i}^{(\text {ext })}$ can be arranged as vectors $\mathbf{P}$ and $\mathbf{E}$ resulting in: $\underline{46}$

$$
\mathbf{P}=\left(\mathbb{I} \omega^{2}-\mathbb{M}\right)^{-1} \mathbb{R} \mathbf{E}
$$

where $\mathbb{M}$ is the dynamical matrix and $\mathbb{R}$ is a diagonal matrix that rescales the external applied field according to local properties:

$$
R_{i, i}=-\varepsilon_{0} \mathscr{N}_{i} \omega_{\mathrm{P} i}^{2} f_{i}
$$

with $f_{i}=\frac{\left[1-\left(\varepsilon_{\infty}-\varepsilon_{m, i}\right)\left(\omega^{2}-i \omega \eta_{i}\right) / \omega_{\mathrm{P} i}^{2}\right]}{\left[\varepsilon_{m, i}+L_{i}\left(\varepsilon_{\infty}-\varepsilon_{m, i}\right)\right]}$.

Because of the cubic dependence of $E$ on $d$, we neglect contributions beyond nearest neighbors,,$\underline{45,46}$ resulting in a tridiagonal $\mathbb{M}$. For NPs belonging to the chain, $(i, j) \neq 0, M_{i, i}=\omega_{\mathrm{SP}}^{2}-\mathrm{i} \eta \omega$ and $M_{i, j}=\omega_{\mathrm{X}}^{2}$, and for the LE-NP, $M_{0,0}=\omega_{\mathrm{SP} 0}^{2}-\mathrm{i} \eta_{0} \omega, M_{0,1}=\omega_{\mathrm{X} 0,1}^{2}$ and $M_{1,0}=\omega_{\mathrm{X} 1,0}^{2}$. The LSP frequencies $\omega_{\mathrm{SP} i}$ and coupling constants $\omega_{\mathrm{X} i j}^{2}$, result:

$$
\begin{gathered}
\omega_{\mathrm{SP} i}^{2}=\frac{\omega_{\mathrm{P} i}^{2} L_{i}}{\left[\varepsilon_{m, i}+L_{i}\left(\varepsilon_{\infty}-\varepsilon_{m, i}\right)\right]}, \\
\omega_{\mathrm{X} i j}^{2}=\frac{\gamma^{T, L} \mathscr{V}_{i} \omega_{\mathrm{P} i}^{2}}{4 \pi \varepsilon_{m} d_{i j}^{3}} f_{i} .
\end{gathered}
$$

For spherical NPs $(L=1 / 3)$ in vacuum $\left(\varepsilon_{m}=1\right)$ within a Drude-Sommerfeld model which completely neglects contribution from bound electrons $\left(\varepsilon_{\infty}=1\right)$, Eqs. ??-?? reduce to the simple form used in several references. $\underline{36}-\underline{41,45,46}$ This shows the meaning of $f_{i}$, which essentially collects the deviations from the ideal model of spherical NP in vacuum with $\varepsilon_{\infty}=1$, not only for $R_{i, i}$ but also for couplings between NPs. Note that these deviations reduce to a constant for $\varepsilon_{\infty} \approx \varepsilon_{m, i}$ and/or 
small excitation frequencies $f_{i}$ (strictly for $\left[\omega^{2}-i \omega \eta_{i}\right] \ll \omega_{\mathrm{P} i}^{2} /\left[\varepsilon_{\infty}-\varepsilon_{m, i}\right]$ ). The form of Eqs. ??-?? not only extends our previous expressions $\underline{\underline{46}}$ to non-spherical NPs but also includes explicitly the interdependencies of $\omega_{\mathrm{X} i j}^{2}, \omega_{\mathrm{SP} i}^{2}$, and $R_{i, i}$ with the parameters of the system, i.e. size, shape, and material of NPs, distances between NPs, and dielectric constants. It should also be mentioned that although this model can be further improved, the different corrections will only add quantitative corrections as long as the essential physics of the problem remains, i.e. a semi-infinite chain with nearest neighbors interactions. $\underline{46,49,53}$ We will analyze this point in more detail in the last section.

In the present work we will consider the case of excitation injection to a semi-infinte homogeneous linear array of NPs, where solely the LE-NP can be different from the rest and the only nonidentical separation is that between the LE-NP and the first NP of the chain. Therefore, there will be two LSP resonances, that of the LE-NP $\omega_{\mathrm{SP} 0}$ and that of the chain's NPs $\omega_{\mathrm{SP}}$. Similarly, the couplings between the chain's NPs will be all the same $\omega_{\mathrm{X}}^{2}$. The couplings between the LE-NP and the first NP of the chain are $\omega_{\mathrm{x} 0,1}^{2}$ and $\omega_{\mathrm{x} 1,0}^{2}$. They are not necessarily equal (see Eq. ??), since they describe quite different situations, i.e. how $P_{1}$ affects $P_{0}\left(\omega_{\mathrm{x} 0,1}^{2}\right)$ and how $P_{0}$ affects $P_{1}\left(\omega_{\mathrm{x} 1,0}^{2}\right)$.

The values of the resonance frequency $\omega_{\mathrm{SP}}$ and the couplings $\omega_{\mathrm{X} i j}^{2}$ can be tuned in different ways. However, one must be careful because they are always interrelated. For example, choosing a different material for the NPs will change both $\omega_{P}$ and $\varepsilon_{\infty}$ and therefore $\omega_{\mathrm{x} i j}^{2}$ and $\omega_{\mathrm{SP}}$. Changing the shape of the NPs will modify $L$ and consequently $\omega_{\mathrm{X} i j}^{2}$ and $\omega_{\mathrm{SP}}$, except for $\varepsilon_{\infty}=\varepsilon_{m}$ in which case it will only modify $\omega_{\mathrm{SP}}$. The volume and distances between NPs will change $\omega_{\mathrm{X} i j}^{2}$. If the material, size and shape of NPs are fixed, the free parameters that can be used for sensing purposes are the dielectric constant of the medium $\varepsilon_{m}$ that alter both $\omega_{\mathrm{SP} i}$ and $\omega_{\mathrm{X} i j}^{2}$, and the distances between NPs that change couplings.

The values of $\omega_{\mathrm{X}}^{2}$ and $\omega_{\mathrm{SP}}$ define the passband which is the interval of frequencies at which an excitation can propagate with only a relatively small decay, given by $\eta$, through the waveguide formed by the linear array of NPs. All excitations with frequencies $\omega$ outside the passband will decay along the chain exponentially and very fast. In the weak damping limit (WDL) $\eta \rightarrow 0$, this passband is given by a simple expression, $\frac{\left|\omega^{2}-\omega_{\mathrm{SP}}^{2}\right|}{\omega_{\bar{X}}^{2}} \leq 2$. This fact shows one of the roles of the 
chain: to determine which frequencies can be propagated. The other role is to perturb the local density of plasmonic states (LDPS) of the LE-NP by pushing the resonance from its uncoupled value at $\omega_{\mathrm{SP} 0}$ to the nearest edge of the passband. $\underline{46.49,53}^{-5}$ This process occurs very abruptly when the resonance is about to cross the passband edge that is when the dynamics of the system changes completely. $\underline{\underline{46.49}}$

This phenomenon can be completely understood in terms of the divergences, or poles, of Eq. ?? in the WDL. $\underline{46}$ Basically there are three different dynamical regimes of interest for excitation transfer: 1) delocalized or resonant state regime, where the maximum of LDPS of the LE-NP falls within the passband and most of the excitation can be transferred to the NP array; 2) localized state regime where there is a very sharp peak in the LDPS outside the passband and most of the excitation remains close to the excitation point; and 3) a transition regime called virtual state where the LDPS presents a non-Lorentzian asymmetric peak just at the passband edge and most of the excitation can be transferred to the waveguide. The transitions between the different dynamical regimes can be obtained analytically in the WDL and assuming frequency independent couplings, where: $\underline{46}$

$$
\alpha=\frac{2 \beta+4 V^{2}-\beta^{2}-1}{4 V^{2}}
$$

gives the resonant-virtual transition and:

$$
\alpha=2 \pm \frac{(1-\beta)}{V}
$$

gives the virtual-localized transition, where $\alpha=\left(\omega_{\mathrm{x} 1,0}^{2} \omega_{\mathrm{x} 0,1}^{2} / \omega_{\mathrm{x}}^{4}\right), \beta=\left(\omega_{\mathrm{SP} 0}^{2} / \omega_{\mathrm{SP}}^{2}\right)$, and $V=\left(\omega_{\mathrm{x}}^{2} / \omega_{\mathrm{SP}}^{2}\right)$. The constant $\alpha$ is the relative coupling between LE-NP and first NP of the chain in units of the coupling in the chain, $\beta$ yields the difference in resonance frequency of the LE-NP with respect to that of a chain's NP, and $V$ measures the strength of the coupling between chain's NPs in units of their own resonance frequency. These formulas, although strictly valid only in the WDL, give an excellent estimation for finites $\eta \mathrm{s} . \underline{46}$

The excitation transferred from the LE-NP to the $m$-th NP of the chain can be calculated exactly 
(in the quasi-static limit and within the nearest-neighbors approximation) by using the formula: $\underline{46}$

$$
P_{m}=\frac{\omega_{\mathrm{X} 1,0}^{2} R_{00} E_{0}}{\omega_{\mathrm{X}}^{2}\left(\left[\omega^{2}-\widetilde{\omega}_{\mathrm{SP} 0}^{2}\right]-\alpha \Pi(\omega)\right)} e^{-m(l \pm i q)},
$$

where $E_{0}$ is the electric field on the LE-NP and the renormalized eigen-frequency $\widetilde{\omega}_{\mathrm{SP}}$ is given by $\widetilde{\omega}_{\mathrm{SP}}^{2}=\omega_{\mathrm{SP}}^{2}-i \eta \omega$. The decay length $l$ and the wavenumber $q$ depend on the self energy $\Pi$ as:

$$
l \pm i q=\ln \left(\omega_{\mathrm{X}}^{2} / \Pi\right)
$$

where $\Pi$ is:

$$
\begin{aligned}
& \Pi(\omega)=\frac{1}{2}\left[\omega^{2}-\widetilde{\omega}_{\mathrm{SP}}^{2}\right]- \\
& \operatorname{sgn}\left(\omega^{2}-\omega_{\mathrm{SP}}^{2}\right) \frac{1}{2} \sqrt{\left[\omega^{2}-\widetilde{\omega}_{\mathrm{SP}}^{2}\right]^{2}-4 \omega_{\mathrm{X}}^{4}} .
\end{aligned}
$$

In all the plots of the next section with use $\left|P_{m}\right|^{2}$ as an indicator of the excitation transferred to the waveguide, with $\omega_{\mathrm{X}}^{2} / \omega_{\mathrm{SP}}^{2}=0.45, \eta / \omega_{\mathrm{SP}}=0.03$, and using $m=8$. We have chosen $m=$ 8 , because around this value the behavior of $\left|P_{m}\right|^{2}$ vs $m$ becomes essentially a small decaying exponential for all $\omega$ within the passband or zero for excitation frequencies outside it. Thus, choosing a bigger value of $m$ would only add a multiplicative factor to the results, i.e. it would not change qualitatively the figures shown.

\section{RESULTS}

In this work, our purpose is to show how to use the sudden change in the dynamical properties of systems tuned around their DPT to device ETPSs. For this purpose, we used the system depicted in 1 as an example. 2 shows the maximum excitation transferred to a NP of the waveguide, $\left|P_{8}\right|^{2}$, enabled by a variation of the excitation frequency $\omega$, at each system configuration. Superposed are the critical values that separates dynamically distinct behaviors calculated in the WDL (Eqs. ?? 


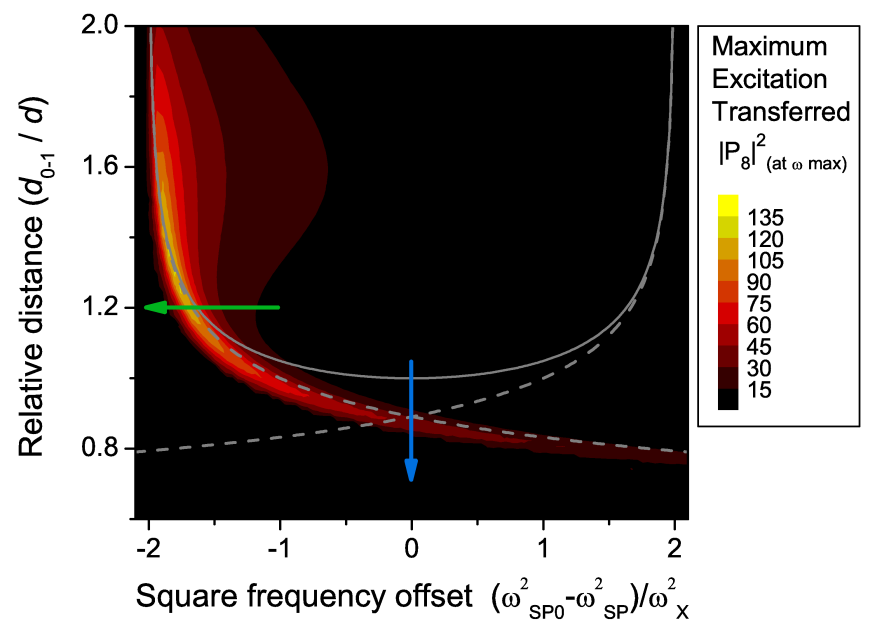

Figure 2: Maximum value, allowed by a variation of the excitation frequency, of the excitation transferred to a NP of the waveguide as function of the system's parameters. gray lines indicate DPTs: the continuous line corresponds to the resonant-virtual DPT and the dashed lines show the virtual-localized transitions.

and ??). As it has been previously reported,,$\underline{46}$ excitation transfer is controlled by the DPT where virtual states are transformed into localized states (gray dashed lines).

Analyzing the phase diagram of 2 it is clear that the strong dependence of excitation transfer to the system's parameters can be used for different forms of sensors. As a first application of this, lets us analyze the potentiality of this plasmonic device as a plasmon ruler. In 2 a vertical displacement corresponds to a variation of the distance between the LE-NP and the chain. The vertical blue arrow indicates an example of a region in the parameters space that can be used for such a purpose. For each point along this arrow the system depicts a different excitation transfer spectrum. Three representative points along this line, at $d_{0-1} / d=0.8,0.9$, and 1.0 , are used in 3-a) to illustrate how the spectrum of excitation transfer changes when the system undergoes a DPT. Note the abrupt change in the spectra induced by a slight change of $d_{01} / d$ : the excitation transfer suddenly increases at $\omega^{2} / \omega_{\mathrm{SP}}^{2} \cong 0.1$ when $d_{01} / d$ varies from 1.0 to 0.9 , and then decreases equally abruptly when $d_{01} / d$ change from 0.9 to 0.8 . This behavior is a consequence of the virtuallocalized DPT, indicated in 2 by the curved gray dashed lines and predicted by Eqs. ?? and ??. One of the interesting aspects of this new form of plasmonic ruler is that the variation of a distance 

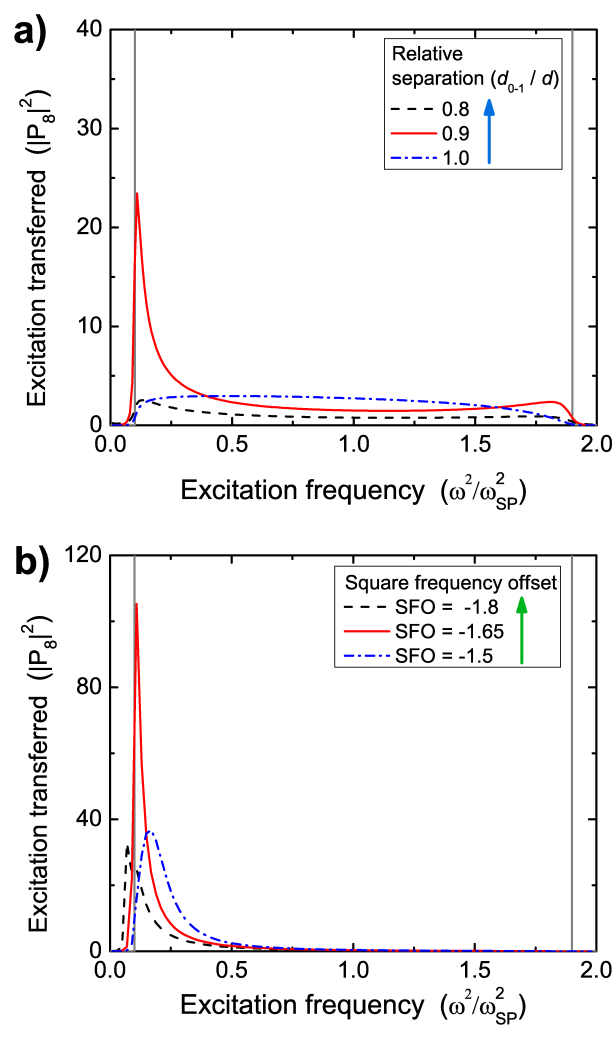

Figure 3: a) Excitation transferred to the 8th NP of the waveguide, $\left|P_{8}\right|^{2}$ in arbitrary units, as a function of $\omega$ for three different values of $d_{0-1} / d$. Each spectrum corresponds to a point along the vertical blue arrow shown in 2 . The value of $\left|P_{8}\right|_{(\text {at } \omega \text { max })}^{2}$ in 2 corresponds here to the maximum value of $\left|P_{8}\right|^{2}$ in a given curve. The two vertical grey lines mark the passband edges of the waveguide in the WDL. b) The same but moving the SFO for fixed $\left(d_{0-1} / d\right)$ along the horizontal green arrow shown in 2 ,

not merely modifies the position of a peak but dramatically changes the whole spectrum, and quite remarkably, before and after the DPT, only negligible signals should be observed independently of the excitation frequency. In this sense, our excitation transfer nanoruler could be used as a robust nanometric standard. This is so, because, in order to observe a difference in the excitation transferred, it is not required a high accuracy in the excitation frequency nor a monochromatic light but keeping the maximum of the excitation frequency close to the lower band edge frequency, in our example at $\omega^{2} / \omega_{\mathrm{SP}}^{2}=0.1$, is a sufficient condition. Furthermore, if the polarization has two components one transversal, $T$, and one longitudinal $L$, this should not interfere in a sensor tuned to work with only one of them. This is so, because the passband of $T$ modes are half of $L$ 
modes, Eq. ??, which imply that regions in the spectrum where DPTs can be observed, should be well separated. Moreover, according to Eqs. ?? and ??, DPTs should occur for different values of system's parameters. Another important aspect is that the detection could be performed in a region encompassing several NPs. This is so, because, except for the first NPs of the chain, the excitation transferred to the chain is an exponential function of NP position $m$, see Eq. ??. Thus, the main feature of this kind of nanorulers is its robust on and off feature, i.e. signals should only be detected when the LE-NP is at a very precise and predetermined distance.
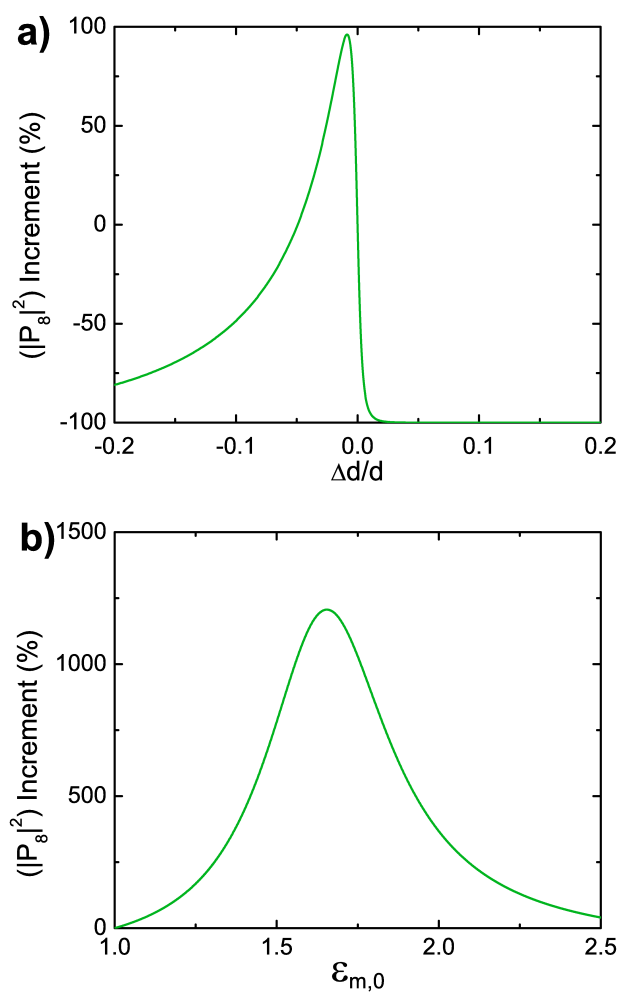

Figure 4: a) Relative increment of the excitation transferred, $\left(|P|^{2}-|P|_{\text {initial }}^{2}\right) /|P|_{\text {initial }}^{2}$, as a function of the relative expansion of the whole system, $\Delta d / d_{\text {initial }}$, for $\omega^{2} / \omega_{\mathrm{SP}}^{2}=0.1$. The initial conditions are $d_{0,1} / d=0.97$ and $\mathrm{SFO}=-1.58$. b) The same but changing the local dielectric constant around the LE-NP, $\varepsilon_{m, 0}$. The initial conditions are $d_{0,1} / d=0.83$ and $\mathrm{SFO}=-1.33$.

ETPS could also be used for sensing other properties, not only as plasmon nanorulers. As shown in 2, the system is also very sensitive to the value of the square frequency offset (SFO = $\left.\left(\omega_{\mathrm{SP} 0}^{2}-\omega_{\mathrm{SP}}^{2}\right) / \omega_{\mathrm{X}}^{2}\right)$ and consequently to $\omega_{\mathrm{SP} 0}^{2}, \omega_{\mathrm{SP}}^{2}$, and/or $\omega_{\mathrm{X}}^{2}$. Increasing the numerator of the SFO or decreasing its denominator, will both move the SFO away from the center of 2 as indicated by 
the horizontal green arrow. 3-b) shows the excitation transfer spectrum for three values of SFO along this green arrow. Similarly to the nanoruler case, the spectrum experiences a significant change when moving the SFO along a DPT, from $S F O=-1.5$ to -1.8 . In order to show how to make profit of this feature we will consider two examples: one in which contraction or expansion of the whole system is measured, and one in which local dielectric constant around the LE-NP is measured. 4-a shows, for a fixed excitation frequency, $\omega^{2} / \omega_{\mathrm{SP}}^{2}=0.1$, the relative increment of the excitation transferred, $\left(|P|^{2}-|P|_{\text {initial }}^{2}\right) /|P|_{\text {initial }}^{2}$, as a function of the relative expansion/contraction of the whole system, which turns our device into an optical strain monitor $\frac{3}{3}$ or even into a temperature sensor, depending on the expansion coefficient of the host material. Note that a relative expansion/contraction of the whole system corresponds to a change in $\omega_{\mathrm{x}}^{2}$ but not in $d_{0-1} / d, \omega_{\mathrm{SP} 0}^{2}$, nor in $\omega_{\mathrm{SP}}^{2}$. Thus it is equivalent to an horizontal displacement in 2, 4-b shows the variation of the relative increment of the excitation transferred as a function of the local dielectric constant around the LE-NP, a property potentially useful for molecular sensing purposes for example. ${ }^{2}$ In this case, a variation of $\varepsilon_{m, 0}$ changes not only $\omega_{\mathrm{SP} 0}^{2}$ but also $\omega_{\mathrm{X} 0,1}^{2}$ and $R_{0,0}$ (Eqs. ??-??). Therefore, it is equivalent to a diagonal displacement in 2 and a rescaling of the external applied field.

\section{RETARDATION EFFECTS AND CONTRIBUTIONS BEYOND NEAREST NEIGHBORS}

The key ingredients from which the phenomenology described so far arise are: dominant nearestneighbors interactions and the semi-infinite character of the system. If this conditions are fulfilled, the results presented above should be applicable to the system under consideration besides quantitative corrections such as shifts of the spectra or the exact position of DPTs in the parameter's phase space. However, it is important to understand how our results are corrected by different factors.

In this section, we will study the effect of interactions beyond nearest-neighbors and retardation effects. In this case, Eq. ?? is still valid but all coupling between NPs should be considered, i.e. $\mathbb{M}$ 
is no longer tridiagonal, and these couplings should be determined by the dipole-induced electric field beyond the quasi-static limit, i.e.:

$$
\vec{E}=\frac{e^{i k d}}{4 \pi \varepsilon d^{3}}\left\{(k d)^{2}(\hat{d} \times \vec{p}) \times \hat{d}+[3 \hat{d}(\hat{d} \cdot \vec{p})-\vec{p}](1-i k d)\right\}
$$

where $k$ is the wavenumber in the dielectric, $k=\omega / v$ (where $v$ is the speed of light in the medium), $\hat{d}$ is the unit vector in the direction of $\vec{d}$ (where $\vec{d}$ is the position of the observation point with respect to the position of the dipole).

As the system consists of a linear array of NPs where the spheroids axes are aligned with respect to the direction of the array, transversal $(T)$ and longitudinal $(L)$ excitations do not mix. Thus, the coupling terms $\omega_{X}^{2}$ of Eq. ?? acquire the form:

$$
\omega_{\mathrm{X} i j}^{2}=\frac{\widetilde{\gamma}_{i, j}^{T, L} \mathscr{V}_{i} \omega_{\mathrm{P} i}^{2}}{4 \pi \varepsilon_{m} d_{i, j}^{3}} f_{i},
$$

where

$$
\begin{aligned}
& \widetilde{\gamma}_{i, j}^{L}=-2\left[1-i k d_{i, j}\right] e^{i k d_{i, j}} \\
& \widetilde{\gamma}_{i, j}^{T}=\left[1-i k d_{i, j}-\left(k d_{i, j}\right)^{2}\right] e^{i k d_{i, j}}
\end{aligned}
$$

Eq. ?? has now to be solved numerically which can be done by using standard methods of linear algebra..$\underline{54}$

The physical system considered in this section corresponds to a linear array of very flat oblate spheroidal NPs of silver $\left(\varepsilon_{\infty}=3.7\right.$, and $\left.\omega_{\mathrm{P}}=9.3 \times 10^{15} \mathrm{rad} / \mathrm{s}\right)$ with the radio of the minor axis equals to $4.5 \mathrm{~nm}$ and the radii of the major axis equal to $46.5 \mathrm{~nm}$ (shape factor $L_{i}=0.86$,). The major to minor axis ratio is about 10 which is large enough to ensure a strong quadrupole quenching. $\underline{55}$ Separation between NPs of the chain is $13.5 \mathrm{~nm}$, from center to center, and they are aligned with the two equal axes perpendicular to the array direction. The external electric field is applied only to the first NP where the direction of $E_{0}^{(e x t)}$ is also perpendicular to the array, i.e. $\gamma=\widetilde{\gamma}_{i, j}^{T}$. Elec- 
tronic damping is calculated using the Matthiessen's rule $\eta=v_{F}\left(1 / l_{\text {bulk }}+C / l_{e f f}\right)$ where the Fermi velocity is $v_{F}=1.38 \times 10^{6} \mathrm{~m} / \mathrm{s}$, the bulk mean free path is $l_{\text {bulk }}=57 \mathrm{~nm}$, we use $C \cong 1$, and the effective mean free path is calculated using $l_{\text {eff }}=4 V / S$ where $V$ is the volume and $S$ the surface of the spheroids. $\frac{56}{}$ For simplicity we consider a dielectric medium with $\varepsilon_{m}=\varepsilon_{\infty}$. Taking into account this, $\eta / \omega_{\mathrm{SP}}$ and $\omega_{\mathrm{X}}^{2} / \omega_{\mathrm{SP}}^{2}$ give 0.032 and 0.41 (for $k d=0$ ) respectively. We use here a slightly smaller coupling than that of the previous section for reasons that will become clear in the context of 5]. As before, the observation point is fixed at the 8th NP, and we use the value of $\left|P_{8}\right|^{2}$ as an indicator of the excitation transferred to the chain. Due to the large interparticle coupling, finite size effects result so important, especially near the lowest band edge, that it is necessary to take very long size of chains. We use 200 NPs, a value large enough to ensure negligible finite size effects.

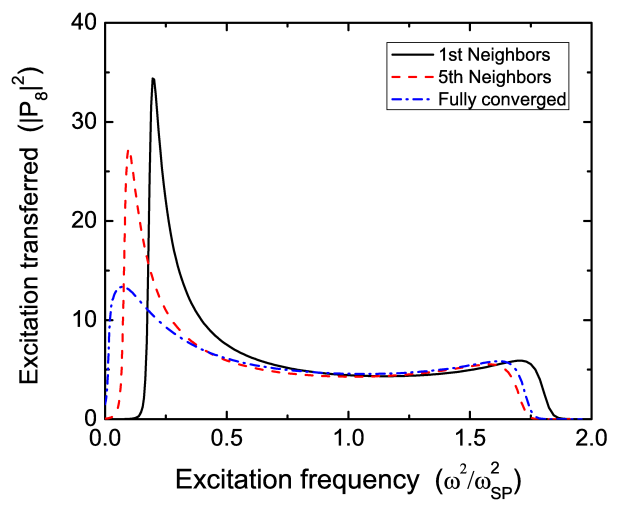

Figure 5: Excitation transferred to the waveguide, $\left|P_{8}\right|^{2}$ in arbitrary units, as function of $\omega^{2} / \omega_{\mathrm{SP}}^{2}$ for $d_{0-1} / d=0.9, \omega_{\mathrm{X}}^{2} / \omega_{\mathrm{SP}}^{2}=0.41$ and $\eta / \omega_{\mathrm{SP}}=0.032$. Different curves show numerical results within the near field approximation for: nearest-neighbor interactions, fifth-nearest-neighbor interactions, and a fully converged calculation. The system is at the DPT for the curve with nearest-neighbor interactions.

5 shows the effect of including interaction beyond nearest-neighbors. The first effect of this is essentially a red-shift and a broadening of the passband. The second effect is that the DPT is also shifted from $d_{0-1} / d=0.9$ to $d_{0-1} / d=0.84$ as can be seen in 6-a. However, it is still present and indeed, as the damping term is proportional to the frequency, the peak is increased. Note that if $\omega_{\mathrm{X}}^{2} / \omega_{\mathrm{SP}}^{2}=0.45$ were used, the peak would disappear from the spectrum as it would 
be located at $\omega^{2}<0$, showing an important effect of higher-order contributions to interactions. In 6-a and $\mathrm{b}$ retardation effects on the DPT are compared. Here, retardation causes nothing else but an increase of the intensity of the peak, in the region of the spectrum where the DPT is observable. This is because, in this region of the spectrum, around $\omega^{2} / \omega_{\mathrm{SP}}^{2}=0.02, d$ is much smaller than $k$ ( $k d \approx 0.05$ ). Of course at higher frequencies, for example at $\omega \approx \omega_{S P}$, this is not the case $(k d \approx 0.4)$ and the behavior of the system is completely beyond the near field approximation.
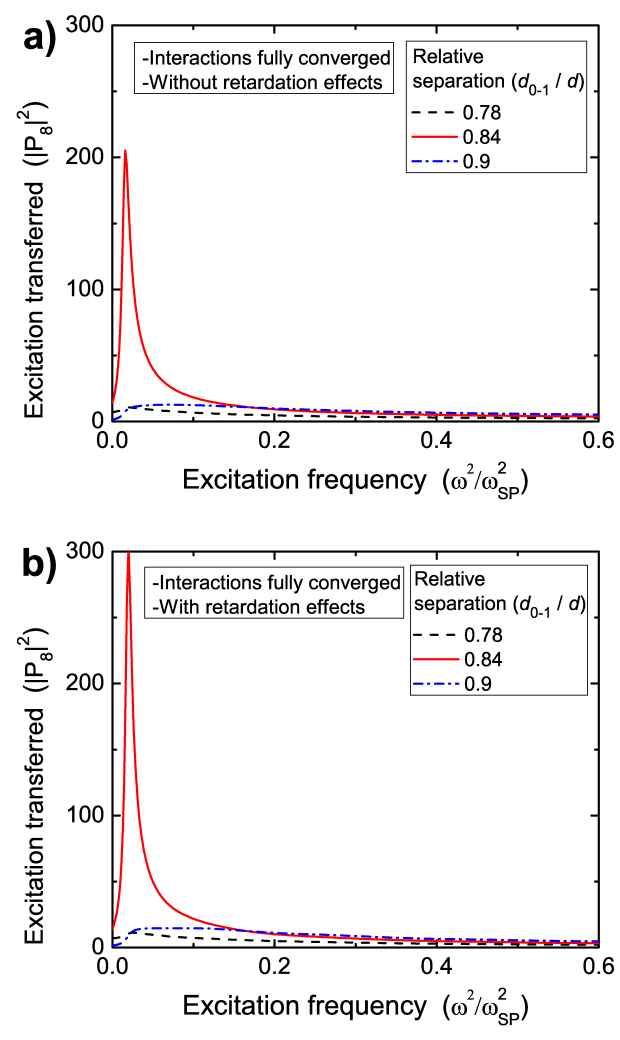

Figure 6: a) Numerical results without retardation effects of the excitation transferred to the waveguide, $\left|P_{8}\right|^{2}$ in arbitrary units, as function of $\omega^{2} / \omega_{\mathrm{SP}}^{2}$ for different values of $d_{0-1} / d$. b) The same but with retardation effects. In both cases all interactions between NPs are considered.

Finally, 7 shows the value of $\left|P_{m}\right|^{2}$ for different NPs of the chain. Note that even after including all contribution to the interaction among NPs and retardation effects, $\left|P_{m}\right|^{2}$ still decays exponentially as predicted by the theory, although the decay rate is different mainly because the passband edge is considerably shifted. 


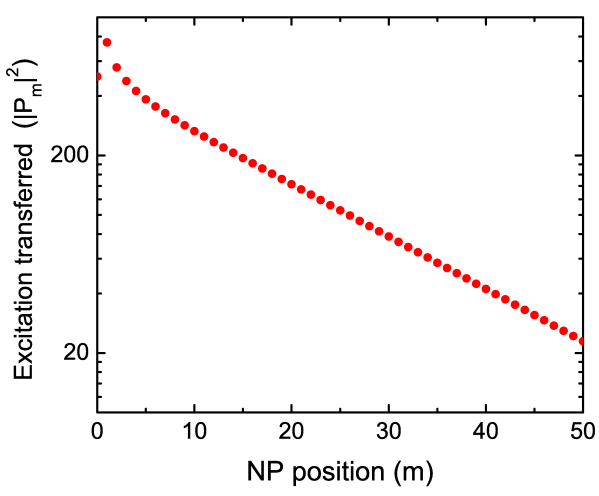

Figure 7: Excitation transferred to a NP of the waveguide, $\left|P_{m}\right|^{2}$ in arbitrary units, as function of its position $m$. Retardation effects as well as all interactions between NPs are considered. Here $d_{0-1} / d=0.84$ and the excitation frequency is $\omega^{2} / \omega_{\mathrm{SP}}^{2}=0.02$, which correspond to the peak of 6-b

\section{CONCLUSIONS}

In this work we have studied the potentiality for sensing purposes of the excitation transferred from a LE-NP to the interior of a semi-infinite NP chain, when the system is very close to its DPT condition. While most plasmonic sensors use the shift of the LSP resonance peaks or the photoluminescence quenching of hybrid plasmonic-fluorophores systems, here we introduce a new working principle. Basically, the idea is to take advantage of the abrupt change in the plasmonic energy transferred when a control parameter is slightly changed around a DPT. We have shown that this kind of sensor has the unique characteristic of having an on-off switching property and a high sensitivity, which opens new possibilities to design plasmonic devices such as plasmonic circuits activated only under certain environmental conditions. We have also addressed the effects of different corrections to the approximations used and have shown that if the system's parameters are chosen properly, the DPT should be observable and therefore useful for the sensing purposes.

\section{References}

(1) Stewart, M. E.; Anderton, C. R.; Thompson, L. B.; Maria, J. ;Gray, S. K.; Rogers, J. A.; Nuzzo, R. G. Chem. Rev. 2008, 108, 494-521. 
(2) Mayer, K. M.; Hafner, J. H. Chem. Rev. 2011, 111, 3828-57.

(3) Halas, N. J.; Lal, S.; Chang, W.-S.; Link, S.; Nordlander, P. Chem. Rev. 2011, 111, 3913-61.

(4) Perassi, E. M.; Canali, L. R.; Coronado, E. A. J. Phys. Chem. C 2009, 113, 6315-6319.

(5) Encina, E. R.; Perassi, E. M.; Coronado, E. A. J. Phys. Chem. A 2009, 113, 4489-4497.

(6) Coronado, E. A.; Encina, E. R.; Stefani, F. D. Nanoscale 2011, 3, 4042-59.

(7) Encina, E. R.; Coronado, E. A. J. Phys. Chem. C 2010, 114, 3918-3923.

(8) Rong, G.; Wang, H.; Reinhard, B. M. Nano letters 2010, 10, 230-8.

(9) Jain, P. K.; Huang, W.; El-Sayed, M. A. Nano Letters 2007, 7, 2080-2088.

(10) Jain, P. K.; El-Sayed, M. A. Phys. Chem. C 2008, 112, 4954-4960.

(11) Tabor, C.; Murali, R.; Mahmoud, M.; El-Sayed, M. A, J. Phys. Chem. A 2009, 113, 1946-53.

(12) Chergui, M.; Melikyan, A.; Minassian, H. J. Phys. Chem. C 2009, 113, 6463-6471.

(13) Funston, A. M.; Novo, C.; Davis, T. J.; Mulvaney, P. Nano Letters 2009, 9, 1651-1658.

(14) Huang, C.-ping; Yin, X.-gang; Kong, L.-bao; Zhu, Y.-yuan J. Phys. Chem. C 2010, 114, 21123-21131.

(15) Liu, C.; Li, B. Q. J. Phys. Chem. C 2011, 115, 5323-5333.

(16) Ben, X.; Park, H. S. J. Phys. Chem. C 2011, 115, 15915-15926.

(17) Haldar, K. K.; Sen, T.; Patra, A. J. Phys. Chem. C 2010, 114, 4869-4874.

(18) Ray, P. C.; Fortner, A.; Darbha, G. K. J. Phys. Chem. B 2006, 110, 20745-8.

(19) Sen, T.; Haldar, K. K.; Patra, A. J. Phys. Chem. C 2008, 112, 17945-17951.

(20) Griffin, J.; Ray, P. C. J. Phys. Chem. B 2008, 112, 11198-201. 
(21) Seelig, J.; Leslie, K.; Renn, A.; Kuhn, S.;Jacobsen, V.;van de Corput, M.; Wyman, C.; Sandoghdar, V. Nano letters 2007, 7, 685-689.

(22) Singh, M. P.; Strouse, G. F. J. Am. Chem. Soc. 2010, 132, 9383-91.

(23) Anker, J. N.; Hall, W. P.; Lyandres, O.; Shah, N. C.; Zhao, J.; Duyne, R. P. Van Nature materials 2008, 7, 442-53.

(24) Hodges, M. D.; Kelly, J. G.; Bentley, A. J.; Fogarty, S.; Patel, I. I.; Martin, F. L.; Fullwood, N. J. ACS nano 2011, 5, 9535-41.

(25) Ando, J.; Fujita, K.; Smith, N. I.; Kawata, S. Nano letters 2011, 11, 5344-8.

(26) Baca, A. J.; Montgomery, J. M.; Cambrea, L. R.; Moran, M.; Johnson, L.; Yacoub, J.; Truong, T. T. J. Phys. Chem. C 2011, 115, 7171-7178.

(27) Maier, S. A. Plasmonics: Fundamentals and Applications; Springer Press: New York, 2007.

(28) Novotny, L.; Hecht, B. Principles of Nano-Optics; Cambridge Press: Cambridge, 2007.

(29) Burin, L.; Cao, H.; Schatz, G.C.; Ratner, M.A. J. Opt. Soc. Am. B 2004, 21, 121-131.

(30) Guillon, M. Opt. Express 2006, 14, 3045-3055.

(31) Zou, S.; Schatz, G. C. Nanotechnology 2006, 17 2813-2820.

(32) Hernández, J. V.; Noordam, L.D.; Bobicheaux, F. J. Phys. Chem. B 2005, 109, 15808-15811.

(33) Backes, T. D.; Citrin, D. S. Phys. Rev. B 2008, 78, 153407.

(34) Gozman, M.; Polishchuk, I.; Burin, A. Phys. Lett. A 2008, 372, 5250-5253.

(35) Maier, S.;Kik, P.; Atwater, H.; Meltzer, S.; Harel, E.; Koel, B.; Requicha, A. Nature Mat. 2003, 2, 229-232.

(36) Citrin, D. S. Nano Letters 2004, 4, 1561-1565. 
(37) Alu, A.; Engheta, N. Phys. Rev. B 2006, 74, 205436.

(38) Malyshev, A. V.; Malyshev, V. A.; Knoester, J. Nano Letters 2008, 8, 2369-2372.

(39) Zou, S.; Schatz, G. C. J. Chem. Phys. 2004, 121, 12606-12.

(40) Abajo, F. J. Rev. Mod. Phys. 2007, 79, 1267-1290.

(41) Markel, V.; Sarychev, A. Phys. Rev. B 2007, 75, 085426.

(42) Park, S. Y.; Stroud, D. Phys. Rev. B 2004, 69, 125418.

(43) Gharghi, M.; Gladden, C.; Zentgraf, T.; Liu, Y.; Yin, X.; Valentine, J.; Zhang, X. Nano letters 2011, 11, 2825-8.

(44) Guo, X.; Qiu, M.; Bao, J.; Wiley, B. J.; Yang, Q.; Zhang, X.; Ma, Y.; Yu, H.; Tong, L. Nano letters 2009, 9, 4515-9.

(45) Brongersma, M. L.; Hartman, J. W.; Atwater, H. A. Phys. Rev. B 2000, 62, R16356-R16359.

(46) Bustos-Marún, R. A.; Coronado, E. A.; Pastawski, H. M. Phys. Rev. B 2010, 82, 035434.

(47) Kottos, T. Nature Physics 2010, 6, 166-167.

(48) Bender, C. M.; Boettcher, S. Phys. Rev. Lett. 1998, 80, 5243-5246.

(49) Dente, A. D.; Bustos-Marún, R. A.; Pastawski, H. M. Phys. Rev. A 2008, 78, 062116.

(50) Rotter, I. J. Phys. A: Mathematical and Theoretical 2009, 42, 153001;

(51) Garmon, S.; Ribeiro, P.; and Mosseri, R. Phys. Rev. E 2011, 83, 23.

(52) Jones, R. Phys. Rev. 1945, 68, 93-96.

(53) Pastawski, H. M.; Medina, E. Rev. Mex. de Fis. 2001, 47 S1, 1-23; and. references therein. 
(54) We used a complex implementation of LU decomposition method. See: Press, W. H.; Teukolsky, S. A.; Vetterling, W. T.; Flannery, B. P. Numerical Recipes in Fortran 77: The Art of Scientific Computing; Cambridge University Press: Cambridge, 1998; Vol. 6.

(55) Kelly, K. L.; Coronado, E. A.; Zhao, L. L.; Schatz, G. C. The Journal of Physical Chemistry B 2003, 107, 668-677.

(56) Coronado, E. A.; Schatz, G. C. J. Chem. Phys. 2003, 119, 3926-3934. 

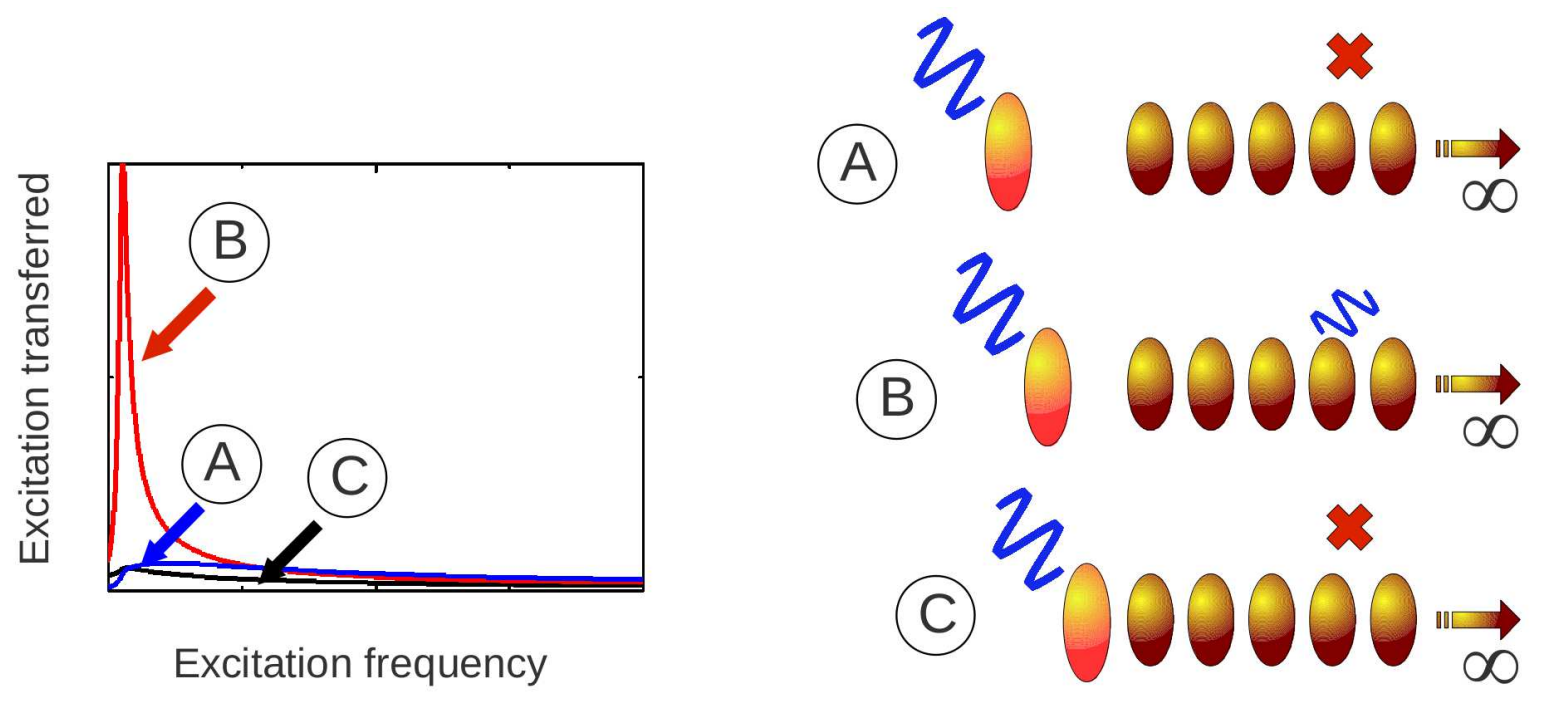

Figure 8: Graphical abstract. 